\title{
From thermal inspection to updating a numerical model of a race bicycle
}

\author{
by G. Steenackers***, J. Peeters*, B. Ribbens*, J. Vanwalleghem**, S. Verwulgen ${ }^{\star}$ and E. Louarroudi* \\ * University of Antwerp, Op3Mech, Salesianenlaan 30, B-2660 Antwerp, Belgium, \\ gunther.steenackers@uantwerpen.be \\ ${ }_{* *}$ Vrije Universiteit Brussel (VUB), Acoustics \& Vibration Research Group (AVRG), B-1000 Brussels, Belgium \\ *** Ghent University, Mechanics of Materials and Structures, 9052 Zwijnaarde, Belgium \\ + University of Antwerp, Product Development, Ambtmanstraat 1, B-2000 Antwerp, Belgium,
}

\begin{abstract}
There are many advantages of using thermography for updating numerical models. These include the fact that it can be performed in-situ, it can cover large areas and it is a quantitative method. In this paper, a numerical model of a bicycle frame will be updated and optimized by the surface temperature distribution captured with pulsed thermography. These results will be compared and benchmarked against FRF measurement data as reference. The chosen temperature decay measurement to be used as reference data will be of key importance. Both updating techniques will be compared with respect to measurement time and accuracy.
\end{abstract}

Keywords: finite element model, pulsed thermography, optimization, Inverse Problem

\section{Introduction}

The study of Pulsed Infrared Thermography (PT) has become an important aspect of non-destructive evaluation technique (NDE) for damage detection in metallic structural elements, as well as CFRP (Carbon Fibre Reinforced Polymer) and GFRP (Glass Fibre Reinforced Polymer) composites [1]. There are many advantages of thermography, these include the fact that no coupling is required, it can be performed in-situ, it can cover large areas and it is a quantitative method. A disadvantage is that there is a limit on the thickness of the structure being monitored [2]. On the other hand, computer simulation and virtual modelling tools are an economical and fast way to determine and simulate material behaviour. The next step in integrating the non-destructive PT tests and virtual modelling is the updating of the FE model [3], [4]. Computer simulations are economically interesting and a fast way to determine and simulate material behaviour and to calculate worst case scenarios. Finite element (FE) analysis has been used as a verification tool in some applications involving infrared thermography [5]. Besides thermography, FE modelling has proven its success in multiple application areas for worst case simulation and quantitative failure detection. In conventional FE model updating procedures, frequency response function measurements and estimated modal parameters are used as reference data to be compared with the calculated output from the FE model [6]. The disadvantages are that there is a limit on the thickness of the structure being monitored [7]. In this paper, a finite element model of a bicycle frame will be updated and optimized by the surface temperature distribution captured with pulsed thermography. These results will be compared and benchmarked against thermal measurement data to be used in an FE updating procedure [8]. The chosen temperature decay measurement to be used as reference data will be of key importance. In this paper, the aim is to update the FE model with respect to the temperature distribution measured by the thermography non-destructive method applied on a carbon race bicycle frame. Both updating techniques will be compared with respect to measurement time and accuracy.

\section{Pulsed thermography}

Pulse thermography (PT) is one of the most popular thermal stimulation method in IR thermography. One reason for this popularity is the quickness of the inspection relying on a thermal stimulation pulse, with duration going from a few ms for high thermal conductivity material inspection (such as metal parts) to a few seconds for low thermal conductivity specimens (such as plastics, graphite epoxy components). Such quick thermal stimulation allows direct deployment on the plant floor with convenient heating sources. Moreover, the brief heating prevents damage to the component (heating is generally limited to a few degrees above the initial component temperature).

In PT experiments, measurement of the temporal evolution of the surface temperature is done by using an infrared (IR) camera to reveal the presence of defects under the surface [2]. Qualitatively the principle is as follow: absorption of energy from the thermal pulse causes surface temperature to change rapidly, a thermal front is thus launched under the surface where it propagates by diffusion. Presence of zones having different thermal properties (defects) with respect to the bulk of material changes the diffusion rate so that defect positions can be seen on the front surface as areas having different temperatures, after the thermal front reaches them. Problems associated with PT are 
generally weak contrasts, detection limited to shallow defects, sensitivity to surface artifacts of the specimen, need to know the localization of a "defect-free" location to compute thermal contrast (e.g. [2], eq. 6.28). On the other hand, as for NDE applications, PT has many known advantages: - non contact, -quick, - ease of deployment, etc.

\section{Measurement Set-up}

The bicycle frame is heated by two heat sources of $1000 \mathrm{~W}$ each. The sources are positioned straight to the wall as shown in Figure 2. The Xenics Gobi camera is placed on the center-to-center distance between both halogen spots. The heating time is 10 seconds and a measured cooling time has been used. There is opted to use a pulsed thermography measurement set-up in order to perform a fast and 'simple' measurement.

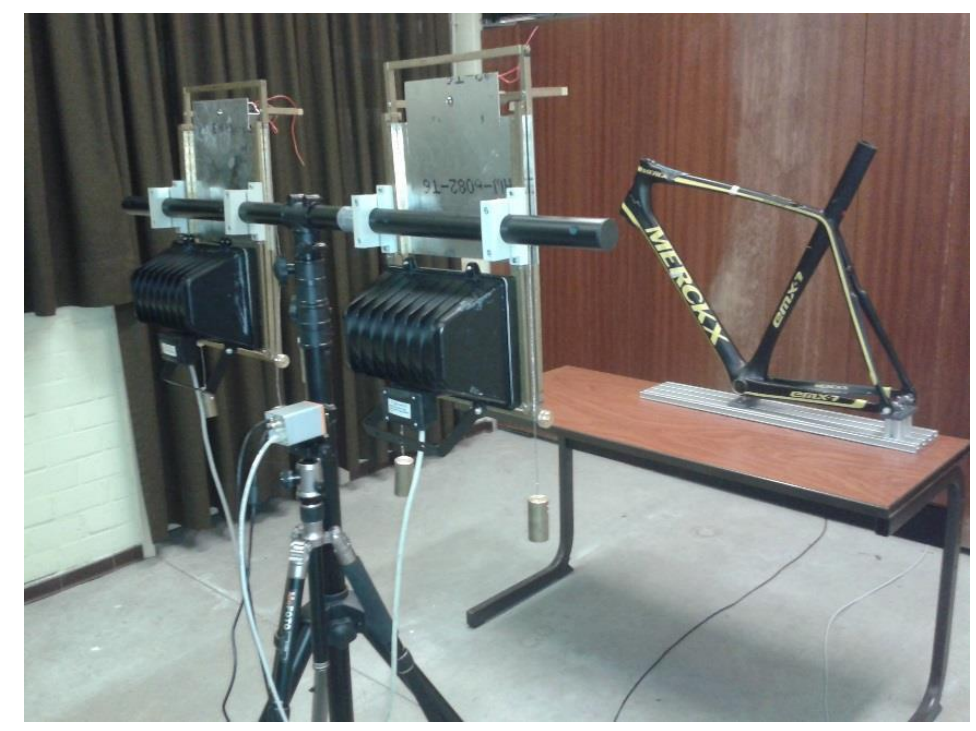

Fig. 1. Experimental setup for IR measurements.

The microbolometer has a field-of-view of $42.6^{\circ}$. This means the camera needs to be placed about $1.3 \mathrm{~m}$ from the bicycle frame in order to capture the complete frame in the camera image. The reflection can be ignored as Germanium has a reflection rate of less than $1 \%$ between a wave length of $7-12 \mu \mathrm{m}$. In order not to have an influence of the wall behind the set-up, the bicycle frame will be placed at a distance of $1 \mathrm{~m}$ from the wall, in order to avoid that the wall heats up due to the light bulb excitation [1,3]. For this reason, the wall reflection will not have an influence on the heat measurements. In addition, the camera will be placed symmetrically between the light bulbs (Figure 1). The halogen light bulbs and microbolometer camera are placed perpendicular to the bicycle frame in order to capture the complete frame at equal distance but also in order to avoid that occurring reflections are not coinciding with the measured bicycle frame.

\section{Measurements}

During the analysis of the images, it was clear that a pulsed heating of 8 seconds is sufficient to heat up the bicycle frame and to make a temperature analysis possible for updating purposes. As already discussed, a pulse thermography measurement procedure is used to perform the measurements. As seen on Figure 2, visualizing a thermal image of the bicycle frame, a contrast over the structure surface is clearly visible and ready for analysis and postprocessing. 


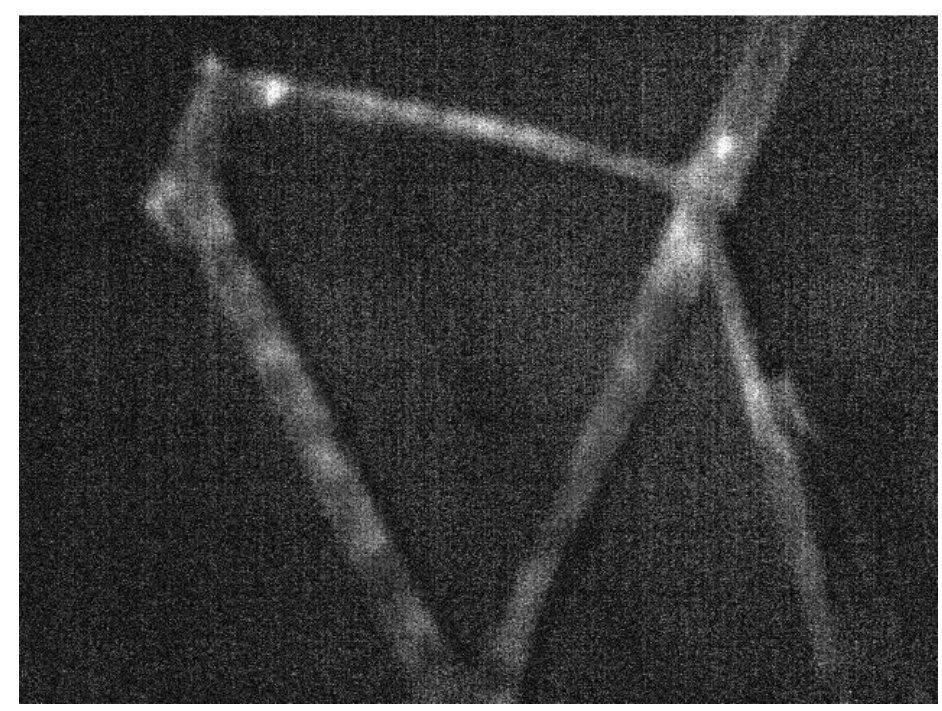

Fig. 2. Measured thermal image of bicycle frame.

\subsection{Measurement parameters}

For the thermal measurements, a Xenics Gig-E Gobi camera is used. The images are captured with a sample frequency of $10 \mathrm{~Hz}$ and a resolution of $640 \times 480$ pixels. The Noise Equivalent Temperature Difference (NETD) of the camera equals $50 \mu \mathrm{K}$ which is a measure for the camera sensitivity.

Different measurement points are chosen over the bicycle frame. These measurement points are visualized in Figure 3, representing a thermal image with a fixed frame number. The measurement points to be used for updating purposes are chosen in the vicinity of the frame where steering stem should be placed. These points are chose in such a way that the updating results are resulting in an accurate prediction of the frame thickness and material properties on this location.

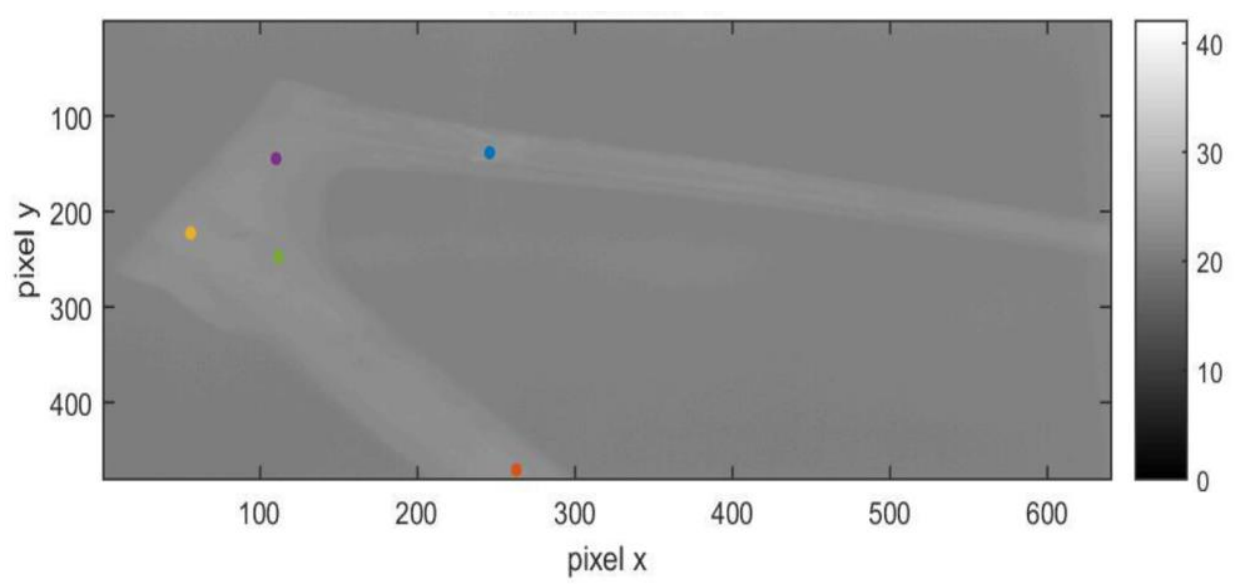

Fig. 3. Thermal image with fixed frame number (frame 8) 


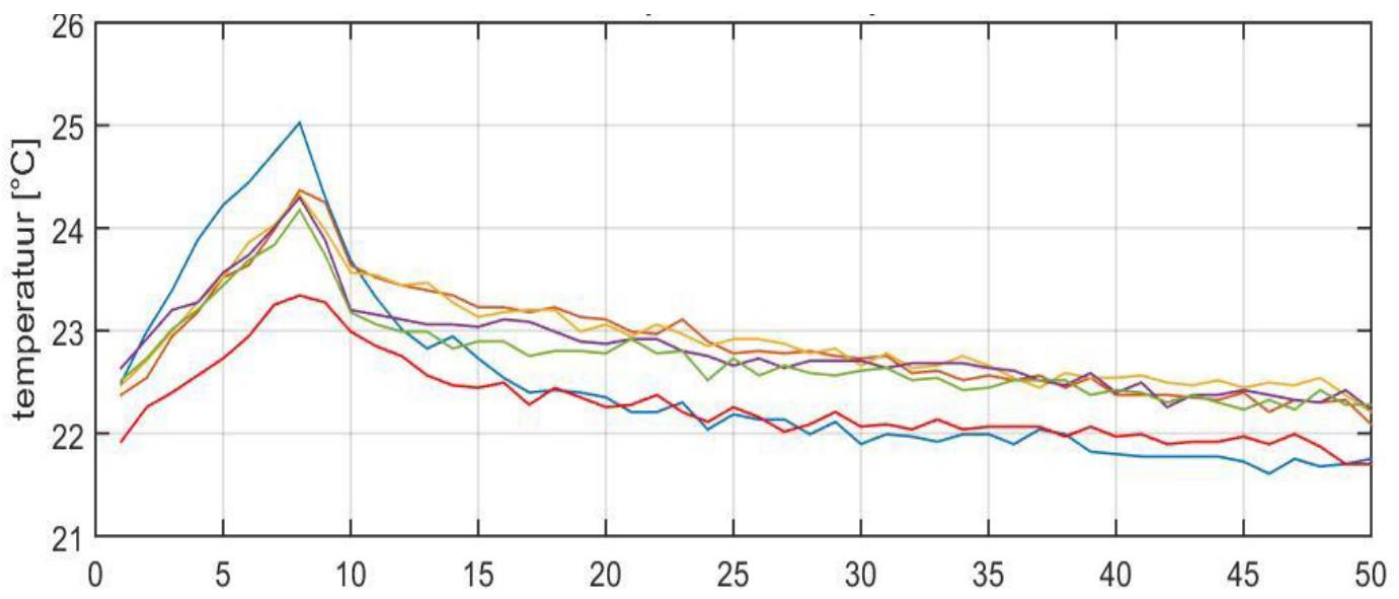

Fig. 1. Temp. profile versus time.

The measured temperature evolution over time (over different measurement frames) is visualized in Figure 4. The heating of the structure surface is clearly visible during the first 8 seconds. Afterwards, the structure's temperature is decreasing over time and the temperature decay in the case of pulsed thermography is measured. The different measurement points over the bicycle frame follow the same temperature evolution over time. The ambient temperature is $22{ }^{\circ} \mathrm{C}$. During the pulsed excitation, the structure is heated a few degrees Celsius. Also on Figure 4, it can be noticed that a difference in material thickness, depending on the location of the measurement points, is resulting in different maximum temperature values. These temperature values will be compared with the simulation results, discussed in Section 5 .

\section{Finite Element model}

The bike frame model (Fig.1) is built in Autodesk Inventor and simulated in Siemens NX. The composite layer is defined in NX Laminate Composites, a module for NX Advanced Simulation that allows to design and analyze laminate composites. The composite model created reflects all geometry and physical aspects of the composite frame. Mesh convergence of the finite element was checked, using 11241 linear tetrahedral elements.

\subsection{Thermal simulation property values}

The finite element model is defined with the material properties of a CFRP composite. De material property values of the four-layer laminate are defined with equivalent density value of $1350 \mathrm{~kg} / \mathrm{m}^{3}$. The used heat capacity is a constant value and thermal conductivity and expansion coefficient are temperature dependent values. The used values in the thermal simulation are listed in Table 1.

\begin{tabular}{lc}
\hline \multicolumn{1}{c}{ Property } & Value \\
\hline Heat source $[\mathrm{W}]$ & 300 \\
$\mathrm{kmat}[\mathrm{W} /(\mathrm{mK})]$ & 0.2 \\
Cpmat $[\mathrm{J} /(\mathrm{kgK})]$ & 1050 \\
rhomat $\left[\mathrm{kg} / \mathrm{m}^{3}\right]$ & 1350 \\
epsmat & 0.8 \\
Start pulse $[\mathrm{s}]$ & 1.5 \\
Tambient $[\mathrm{K}]$ & 295.15 \\
\hline
\end{tabular}

Table. 1. Thermal simulation property values 


\subsection{1/qirt.2016.123}

\subsection{Mesh}

For the surface of the light bulbs, a 2D QUAD4 surface mesh is chosen with element size $=10 \mathrm{~mm}$ and thickness $=1 \mathrm{~mm}$. The light bulbs are modeled in such a way that the temperature difference is negligible over the bulb model surface. For the frame meshing itself, a 3D Tetra volumetric mesh is chosen with element size equal to $5 \mathrm{~mm}$.

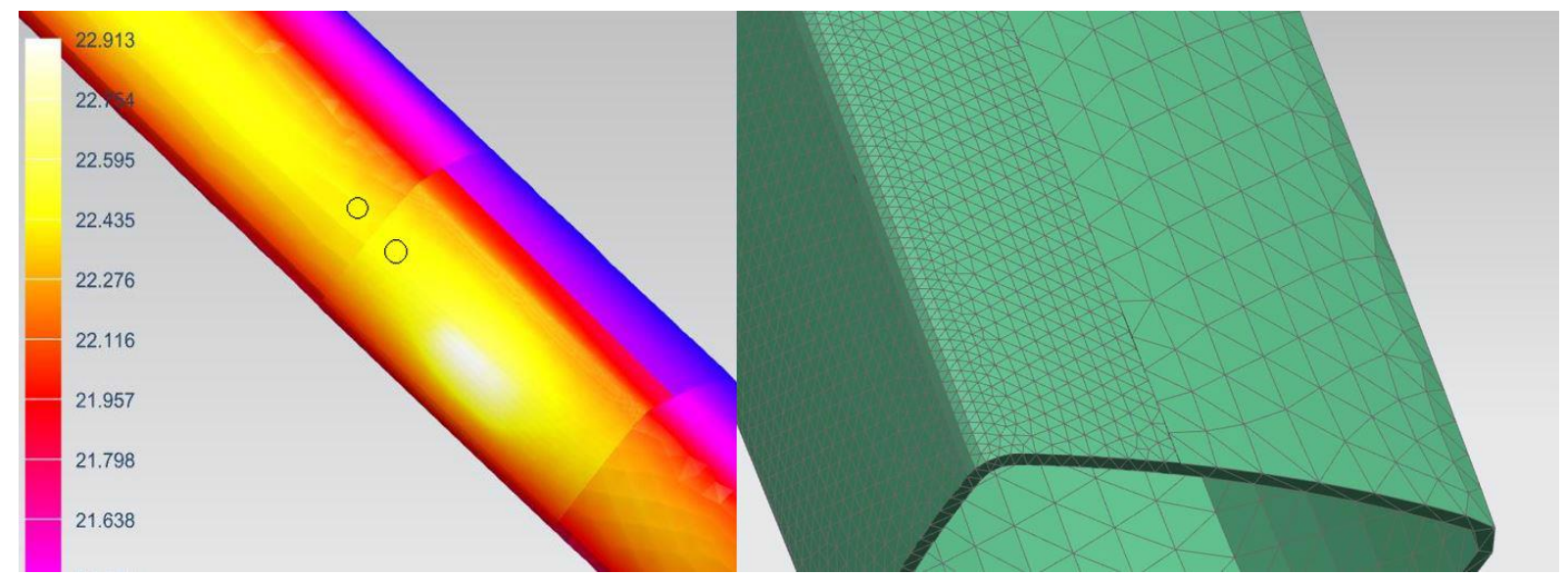

Fig. 5. Bicycle frame mesh and simulated temperature distribution.

\subsection{Heat flux light bulbs}

A power of $1000 \mathrm{~W}$ is defined for the heat sources. As the structured is excited by a pulsed block wave in the case of pulse thermography measurement process, the same heating process is simulated. However, in order to achieve a perfectly flat block rectangular block wave in the heat simulation, a temperature constraint is defined in combination with a variable pulse length in order to optimize the heating of the bicycle frame. An initial excitation pulse length of $8 \mathrm{~s}$ is initially used (Fig. 6). In order to transmit the thermal energy onto the bicycle frame, radiance but also convection is defined in the thermal simulation.

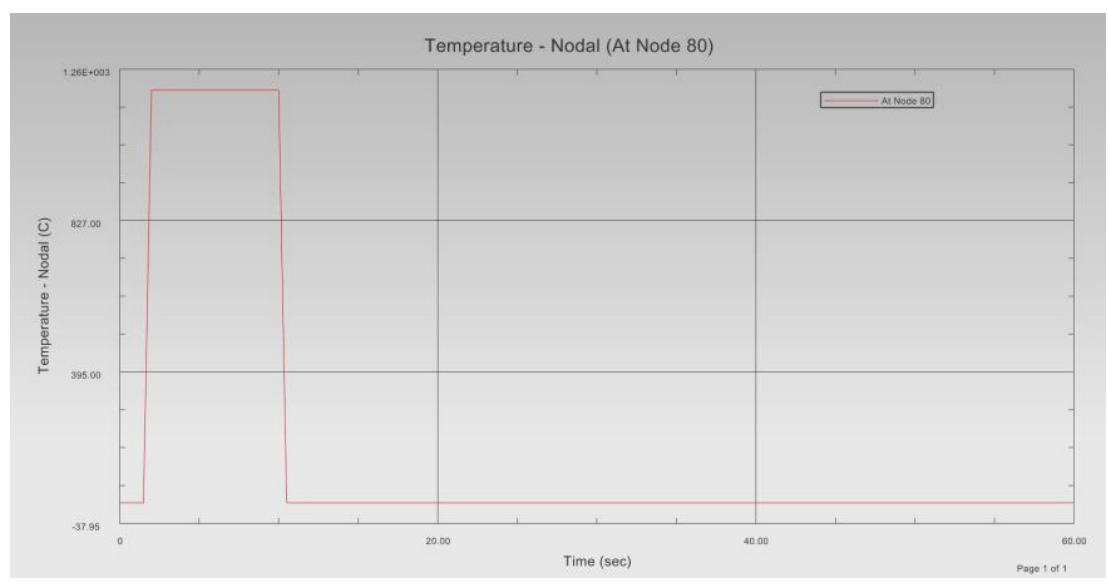

Fig. 6. Heat excitation pulse of flash light bulb during defined in the FE simulation. 


\begin{tabular}{cc}
\hline Parameter & Range \\
\hline Thickness $[\mathrm{mm}]$ & $1-10$ \\
Heat transfer coefficient $[\mathrm{W} / \mathrm{mK}]$ & $2-100$ \\
Pulse duration $[\mathrm{s}]$ & $2-10$ \\
\hline
\end{tabular}

Table. 2. Parameter ranges during optimization routine

\section{Optimization results}

For the FE updating algorithm an adaptive response surfaces optimization approach will be used [8] where the experimental data is used as target for the FE model. The listed parameters (in Table 2) of the frame are the parameters that will be optimized. Figure 1 and Figure 4 present the experimental and numerical results of the first measurements. Figure 4 shows the temperature evolution when applying a pulsed heat source. This difference will be reduced in the optimization algorithm over a time interval. The finite element simulation approximates the experimental setup, which is used to simulate with the correct properties. The updated FE models could be used to assist NDT methods in damage localization and detection.

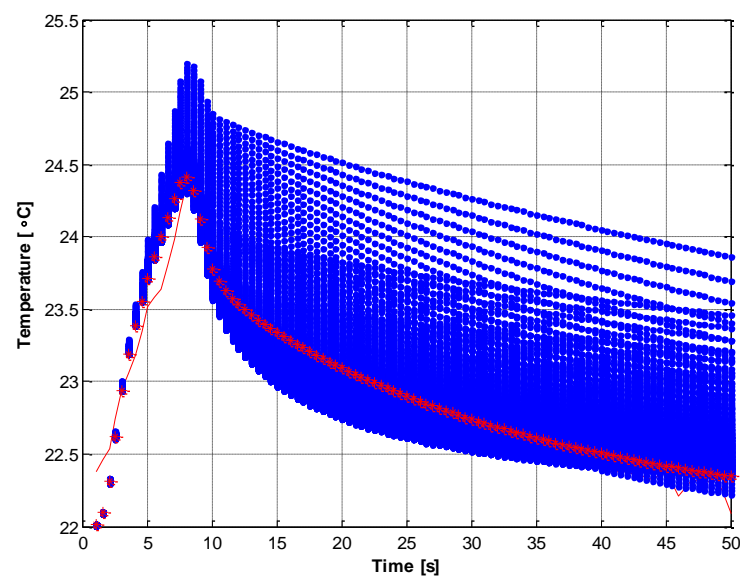

(a)

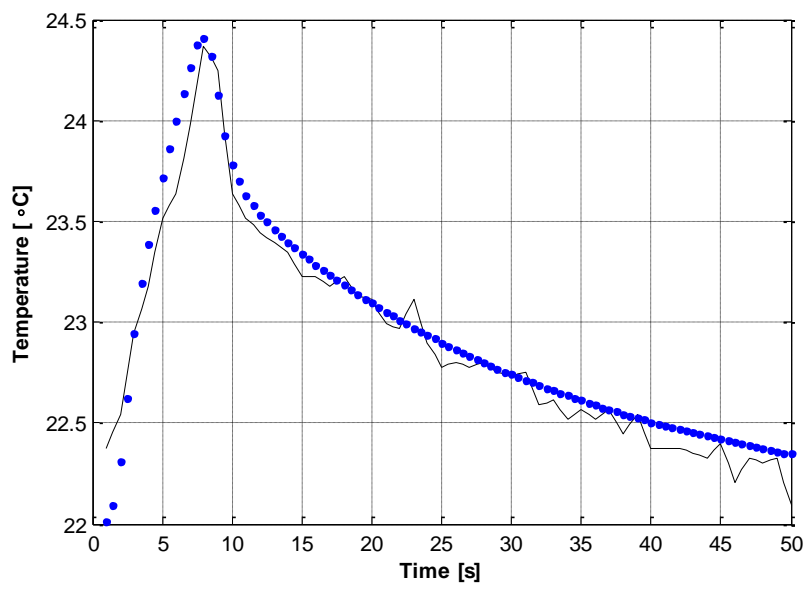

(b)

Fig. 7. Updating of simulated temperature evolution over time with measured decay (full line) and optimized temperature decay (dotted line).

The evolution of the simulated temperature evolution over time is visualized in Figure $7(\mathrm{a})$ with the red dotted line the temperature decay optimally representing the measured temperature decay, plotted in full red line. Both curves are also visualized in Figure $7(\mathrm{~b})$. In this figure it can be clearly seen that the dotted line (simulated temperature) represents the measure temperature decay (full line) very well. The squared error equals a value of 1.4826 . The optimized set of parameter values is listed in Table 3.

\begin{tabular}{lc}
\hline \multicolumn{1}{c}{ Parameter } & Optimal Value \\
\hline Thickness [mm] & 3 \\
Heat transfer coefficient $[\mathrm{W} /(\mathrm{m} . \mathrm{K})]$ & 50 \\
Pulse duration [s] & 8 \\
\hline
\end{tabular}

Table. 3. Optimal parameter values after updating finite element model with temperature measurement. 


\section{CONCLUSIONS}

In this paper, a numerical model of a bicycle frame is updated and optimized by the surface temperature distribution captured with pulsed thermography. From initial updating and optimization results it can be concluded that a temperature decay measurement can be used as reference data in order to determine the correct set of updating parameters. In this case study, the most important parameter value to be identified is the local thickness value of a composite bicycle frame. By making use of a finite element model characterizing and simulating the thermal measurement, one is able to identify the correct thickness, excitation period and convection coefficient.

\section{ACKNOWLEDGEMENTS}

This research has been funded by the University of Antwerp and the Institute for the Promotion of Innovation by Science and Technology in Flanders (IWT) by the support to the TETRA project 'Smart data clouds' with project number 140336. Furthermore, the research leading to these results has received funding from Industrial Research Fund FWO Krediet aan navorsers 1.5.240.13N. The authors also acknowledge the Flemish government (GOAOptimech) and the research councils of the Vrije Universiteit Brussel (OZR) and University of Antwerp (fti-OZC) for their funding.

\section{REFERENCES}

[1] M. Suša, S. Švaić, and I. Boras, "Pulse thermography applied on a complex structure sample: comparison and analysis of numerical and experimental results," in IV Pan American Conference for Non Destructive Testing 2007, 2007.

[2] G. Busse, "Techniques of Infrared Thermography: Part 4. Lock-in thermography," in Nondestructive Handbook, Infrared and Thermal Testing, Volume 3, 3rd editio., X. P. Maldague and P. O. Moore, Eds. Columbus, Ohio: ASNT Press, 2001, p. 718.

[3] J. Peeters, G. Steenackers, B. Ribbens, G. Arroud, and J. Dirckx, "Finite element optimization by pulsed thermography with adaptive response surfaces .," in QIRT2014 Conférence Bordeaux, 2014, p. 10.

[4] J. Peeters, G. Arroud, B. Ribbens, J. J. J. Dirckx, and G. Steenackers, "Updating a finite element model to the real experimental setup by thermographic measurements and adaptive regression optimization," Mech. Syst. Signal Process., vol. 64-65, pp. 428-440, 2015

[5] P. Chaudhuri, P. Santra, S. Yoele, A. Prakash, D. C. Reddy, L. T. Lachhvani, J. Govindarajan, and Y. C. Saxena, "Nondestructive evaluation of brazed joints between cooling tube and heat sink by IR thermography and its verification using FE analysis," NDT E Int., vol. 39, no. 2, pp. 88-95, 2006.

[6] M. Friswell and J. E. Mottershead, Finite element model updating in structural dynamics, vol. 38. Springer Science \& Business Media, 1995.

[7] X. P. Maldague, Theory and Practice of Infrared Technology for Nondestructive Testing. Springer; Softcover reprint of the original 1st ed. 1993 edition (20 Nov. 2011), 2001.

[8] G. Steenackers, F. Presezniak, and P. Guillaume, "Development of an adaptive response surface method for optimization of computation-intensive models," Comput. Ind. Eng., vol. 57, no. 3, pp. 847-855, 2009. 